\title{
Anodal direct current stimulation in the healthy aged: Effects determined by the hemisphere stimulated
}

\author{
Jodie Marquez $^{\mathrm{a}, \mathrm{b}, *}$, Alexander Conley ${ }^{\mathrm{b}, \mathrm{d}}$, Frini Karayanidis ${ }^{\mathrm{b}, \mathrm{d}}$, Jim Lagopoulos $^{\mathrm{c}}$ and Mark Parsons $\mathbf{s}^{\mathrm{a}, \mathrm{b}, \mathrm{e}}$ \\ ${ }^{\mathrm{a}}$ Faculty of Health and Medicine, University of Newcastle, Callaghan, NSW, Australia \\ ${ }^{\mathrm{b}}$ Hunter Medical Institute, New Lambton, NSW, Australia \\ ${ }^{\mathrm{c}}$ Brain and Mind Institute, Sydney University, Sydney, NSW, Australia \\ ${ }^{\mathrm{d}}$ Faculty of Science and IT, University of Newcastle, Callaghan, NSW, Australia \\ ${ }^{\mathrm{e}}$ Department of Neurology, John Hunter Hospital, New Lambton, NSW, Australia
}

\begin{abstract}
.
Purpose: Research popularity and scope for the application of transcranial direct current stimulation have been steadily increasing yet many fundamental questions remain unanswered. We sought to determine if anodal stimulation of either hemisphere leads to improved performance of the contralateral hand and/or altered function of the ipsilateral hand, or affects movement preparation, in older subjects.

Method: In this cross-over, double blind, sham controlled study, 34 healthy aged participants (age range 40-86) were randomised to receive 20 minutes of stimulation to either the dominant or non-dominant motor cortex. The primary outcome was functional performance of both upper limbs measured by the Jebsen Taylor Test and hand grip strength. Additionally, we measured motor preparation using electrophysiological (EEG) recordings.

Results: Anodal stimulation resulted in statistically significantly improved performance of the non-dominant hand $(p<0.01)$ but did not produce significant changes in the dominant hand on any measure $(p>0.05)$. This effect occurred irrespective of the hemisphere stimulated. Stimulation did not produce significant effects on measures of gross function, grip strength, reaction times, or electrophysiological measures on the EEG data.

Conclusion: This study demonstrated that the hemispheres respond differently to anodal stimulation and the response appears to be task specific but not mediated by age.
\end{abstract}

Keywords: tDCS, upper limb function, transcranial direct current stimulation, ageing

\section{Introduction}

Transcranial direct current stimulation (tDCS) has been highlighted as a non-invasive method of modulating brain function. It has been consistently shown in

*Corresponding author: Jodie Marquez, Faculty of Health and Medicine, University of Newcastle, Callaghan, NSW, 2305, Australia. Tel.: +61 2 49212041; Fax: +61 2 49217479; E-mail: Jodie.Marquez@newcastle.edu.au. healthy young adults that cortical activity can be temporarily altered by applying a weak continuous current between two electrodes positioned on the scalp. The effects depend on the position and polarity of the electrodes; specifically brain activity is increased by anodal stimulation and decreased by cathodal stimulation. The published beneficial effects are diverse and include improved: visuo-motor performance (Antal, Kincses et al., 2004), implicit learning (Nitsche, Schauenburg et al., 2003, Kincses, Antal et al., 2004, Kang and 
Paik, 2011), procedural learning (Tecchio, Zappasodi et al., 2010, Stagg, Jayaram et al., 2011), working memory (Zaehle, Sandmann et al., 2011), reaction time (Nitsche, Schauenburg et al., 2003), fine motor skills (Vines, Nair et al., 2006, Vines, Cerruti et al., 2008, Reis, Schambra et al., 2009), functional performance (Boggio, Castro et al., 2006), and muscle endurance (Cogiamanian, Marceglia et al., 2007). Because it is portable, relatively inexpensive, and safe, there is a growing interest in utilizing tDCS in the management of several disease conditions which produce cognitive and movement dysfunction.

There is a paucity of research evaluating the effects of tDCS in the aged. The need for further research in this population is two-fold. Firstly, ageing is associated with an increased prevalence of disease conditions such as Stroke, Parkinson's Disease and Alzheimer's Disease. Extrapolating results from studies in young adults to patients with disease conditions prevalent in aged populations may not be valid given that both cortical structure and function change with age (Spreng, Wojtowicz et al., 2010). Ageing leads to alterations in the excitability of the motor cortex (Oliviero, Profice et al., 2006) which may impact on the effects of cortical stimulation. Furthermore, the comparison of movement related outcomes between different age groups may be invalid as studies have shown that the kinematics of limb movement is altered with age such that movement patterns become more rigid and reaction times are increased (Bennett and Castiello, 1995). Secondly, healthy aging is associated with a successive decline in cognitive and motor abilities which impair independent functioning (Burke and Barnes, 2006). It has been speculated (Zimerman and Hummel, 2010) that noninvasive brain stimulation may be able to ameliorate the decline in this population with obvious potential social and financial benefits.

To our present knowledge, only two clinical studies have examined the effects of anodal stimulation in the healthy aged. Hummel et al. (2010) and Zimerman et al. (2013) examined the effects of anodal tDCS applied over the motor cortex of older adults and demonstrated that upper limb functional performance could be improved in a manner consistent with the findings of younger patients. While these results are promising, they are limited in terms of generalisation as they only assessed the effects of dominant cortex stimulation on dominant hand function. As anodal stimulation is thought to increase excitation of the underlying cortex it is feasible that it may simultaneously decrease contralateral excitation via transcallosal inhibition, thus potentially impairing ipsilateral hand function. Similarly, behavioural effects of the cathode over the contralateral prefrontal cortex cannot be ruled out (Zimerman et al., 2013). Thus these preliminary positive findings warrant replication and more extensive study.

In this study, we used a double blind randomised controlled design to examine whether anodal stimulation of either hemisphere leads to improved performance of the contralateral hand and/or altered function of the ipsilateral hand. In addition, we examined movement preparation and selection using a cued go/nogo task while recording both behavioural and electroencephalography (EEG) data. Electrophysiologically, motor preparation is indexed by the contingent negative variation (CNV) component, indicating the level of readiness to respond to a predicted target (Leuthold, Sommer et al., 2004) and has been linked to the level of excitation in the supplementary motor cortex (Luck, 2005). Hence we can examine the effect of anodal tDCS on movement preparation by examining response times and CNV amplitude to prepared and unprepared responses following active and sham stimulation.

\section{Methods}

\subsection{Subjects}

Subjects were recruited from the Hunter Medical Research Institute volunteer register. We included 34 right handed subjects over the age of 40 years with normal physical and neurological functioning. The time in life when brain ageing begins is undefined, however genetic studies suggest measureable decline after the age of 40 years (Lu, Pan et al., 2004). Left handed subjects were excluded as laterality in the motor hand function tests might not be present in these subjects (Ozcan, Tulum et al., 2004). Hand dominance was determined using the modified Edinburgh Handedness Inventory (Dragovic, 2004). Other exclusion criteria were: reduced cognitive functioning (i.e. a score of 24 or less on the Montreal Cognitive Assessment scale (Nasreddine, Phillips et al., 2005), reported history of neurological disease or muscular dysfunction, psychiatric illness, use of CNS-acting medication, pregnancy, metal implants in the cranium or upper torso, unstable medical conditions, or skin lesions on the scalp. 


\subsection{Study design}

Participants were allocated via computer generated randomization on a 1:1 ratio to one of two treatment orders: sham/tDCS or tDCS-sham. They were then randomized to receive the intervention to either their dominant or non-dominant hemisphere. During each session, the assessment of function and strength was conducted prior to and immediately after the intervention. These assessments included the Jebsen Taylor Hand Function Test (JTT) - a validated timed test of seven functional tasks such as manipulating objects, writing, turning pages etc. (Jebsen et al., 1969) followed by key grip and pinch grip strength - maximal strength as measured by dynamometer. Response processes were assessed using a cued go/nogo paradigm during which electrophysiological (EEG) data were recorded. This task included separate blocks of directional and non-directional cue blocks. All trials began with a small fixation cross which was followed after $500 \mathrm{~ms}$ by the cue onset. The cue-target interval was $1500 \mathrm{~ms}$ and the target stayed on the screen for $1000 \mathrm{~ms}$. The cue consisted of two white arrows pointing in opposite directions $(<>)$ for non- directional trials, and validly predicted the timing of target onset. The target was two green directional arrows in bold («or») that indicated the response hand. For directional trials, the cue consisted of two white arrows (»or «) that validly predicted the direction of the target arrows and therefore the required response. For $70 \%$ of trials the target was the predicted directional green arrows, identical to those in the non-directional cue condition. On the remaining $30 \%$ of trials the target was a red cross ( $\mathrm{x}$ ) indicating that the prepared response must be witheld (e.g. nogo trial). Participants were instructed to prepare a motor response with the hand indicated by the cue but wait until the target to emit the prepared response (go target) or withhold the response (nogo target). Participants completed three brief practice blocks prior to the intervention, and the task consisted of three blocks of the directional cues and two blocks of non directional cues. The total duration of this testing was 38 minutes and it occurred directly after the administration of the post-stimulation functional measures.

Both assessors and subjects were blinded to the type of intervention (sham/anodal tDCS) which was applied in a cross-over sequence with a fixed washout period of three weeks. At the conclusion of each session, participants were asked to complete a questionnaire to indicate whether they believed they had received the active treatment or the sham condition and to document any adverse effects.

\section{3. $t D C S$}

Anodal tDCS was delivered using a commercially available, programmable, direct current stimulator (neuroConn DC-stimulator). Two saline-soaked electrodes $\left(35 \mathrm{~cm}^{2}\right)$ were placed on the scalp with the anode positioned in the region over the primary motor cortex (centred on $\mathrm{C} 3$ for the dominant hemisphere and $\mathrm{C} 4$ for the non-dominant hemisphere) using the 1020 electroencephalogram system. The correspondence of these surface areas to the primary motor cortices has been confirmed in neuroimaging studies (Herwig, Satrapi et al., 2003). The cathode was positioned on the contra lateral supraorbital region. This electrode arrangement is the most typically reported configuration for stimulating the cortical region which represents hand function (Floel and Cohen, 2010, Hummel, Heise et al., 2010).

A current of $1 \mathrm{~mA}$ was applied for 20 minutes. The stimulator was programmed to ramp up the current over several seconds to minimize discomfort. The participants were informed that they could expect to experience a tingling (but not unpleasant) sensation under the electrodes which would rapidly dissipate such that there was little or no physical perception of stimulation after approximately 2 minutes. The set up for the sham condition was identical with the stimulator programmed to turn off after the initial 30 seconds. This has previously been shown to be an effective sham condition which is indistinguishable from the true intervention (Hummel, Celnik et al., 2005, Gandiga, Hummel et al., 2006, Nitsche, Cohen et al., 2008). As several studies have demonstrated that the physiological state of the subject during stimulation can impede the effects of tDCS (Antal, Terney et al., 2007, Quartarone Morgante, 2004), subjects were instructed to sit quietly during the stimulation to avoid interference from cognitive or physical activity.

\subsection{Data analysis}

Demographic and disease characteristics of participants were compared between the intervention and control groups at baseline using Chi-square tests or Fisher's exact test for characteristics with a small number of participants in some cells of cross-tabulations. 
The main functional outcome measure was the difference between a subject's total score on the JTT before and after treatment for each stimulation condition. We also analysed the subscores of fine motor tasks (items 1 to 4 ) and gross motor tasks (items 5 to 7 ) on the JTT and both grip measures The mean and $95 \%$ confidence intervals are reported for each intervention group (sham, tDCS) at each time point. The five motor function measures (total JTT score, gross and fine motor subscales of the JTT, and the two pinch-grip measures) were analysed using a four-way mixed-design analysis of variance (ANOVA), with one between subjects factor: Hemisphere of intervention (dominant, non-dominant) and three within subjects factors: Stimulation condition (anodal tDCS, sham), Hand (left, right) and Time (pre-, post-intervention). It is important to note that in these analyses an effect of anodal tDCS is represented in a significant stimulation $\times$ time interaction, i.e. greater improvement in responding from pre-intervention to post -intervention scores for active as compared to sham stimulation. Behavioural go/nogo task data were also analysed using a four-way mixed-design ANOVA with Hemisphere, Stimulation, Hand and Cue (directional, non-directional). To control for the effect of age on any significant effects we also re-ran these analyses including participants' age as a covariate. Note that in these analyses, an effect of anodal tDCS is represented in a significant stimulation main effect or interaction with other factors, as there was no pre-intervention assessment on the go/nogo task.

The EEG was continuously sampled at $2048 \mathrm{~Hz} /$ channel reference free using a BioSemi ActiView II system. Activity was recorded using a standard 64-channel montage as well as left and right mastoids, the supraorbital and infra-orbital electrodes of each eye, and the two lateral orbital electrodes. Subjects were seated in front of a computer screen in a customised chair with a push button in each of the armrests. Continuous EEG files were re-referenced to average mastoids, and filtered at $0.02-30 \mathrm{~Hz}$. A $50 \mathrm{~Hz}$ notch filter was used to remove line noise. EEG data were processed and analysed using EEG Display 6.3.12 (W.R. Fulham). EEG epochs were extracted from $500 \mathrm{~ms}$ before cue onset to $1000 \mathrm{~ms}$ after target onset and were over a $200 \mathrm{~ms}$ interval prior to onset of the fixation cue. Mean amplitude of the late $\mathrm{CNV}$ was measured at the vertex $(\mathrm{Cz})$ over 1300-1500 ms after cue onset and was analysed using the same four-way mixed models ANOVA as the behavioural data.

\section{Results}

\subsection{Participant characteristics}

Demographic and clinical characteristics assessed included age, gender and cognition (MoCA). Average age was 61 years (range 41-86) with 19 males and 15 females. Age and gender were evenly distributed between the groups defined by the side of the cortex stimulated $\left(t=0.61, P=0.54 ; \chi^{2}=1.94, P=0.16\right)$. All MoCA scores were within normal limits $($ mean $=27.9$, range 24-30) therefore no subjects were excluded from the analyses (Table 1). At baseline, all measures were consistent with age matched normative data (Jebsen, Taylor et al., 1969)

\subsection{Functional motor measures}

Total JTT: As shown in Fig. 1 (left), response time did not differ as a function of Hemisphere of intervention $(p>0.1)$. JTT was completed faster with the right than with the left $(F(1,32)=455.09, p<0.001)$. It was also completed faster post-intervention compared to pre-intervention $(F(1,32)=26.38, p<0.001)$, indicating a significant practice effect. The significant interaction between Hand and Time $(F(1,32)=18.7$, $p<0.001)$ indicates a greater improvement with practice for the left hand.

There was a significant interaction between Stimulation and Time $(F(1,32)=4.31, p=0.046)$, indicating more improvement following anodal tDCS compared to sham. This improvement was significantly greater for the left compared to the right hand (Stimulation $\times$ Time $\times$ Hand: $F(1,32)=7.9$, $p=0.008)$. As shown in Fig. 1 (left), this left hand

Table 1

Demographic and baseline characteristics

\begin{tabular}{lc}
\hline Characteristic & \\
\hline Gender & Males $19(56 \%)$ \\
Age & $61.4 \pm 12.2$ \\
MoCA & $27.9 \pm 2.0$ \\
JTT dominant hand & $43.2 \pm 7.7$ \\
JTT non-dominant hand & $67.2 \pm 13.5$ \\
Key grip dominant hand & $18.2 \pm 5.6$ \\
Key grip non-dominant hand & $17.7 \pm 4.7$ \\
Tip grip dominant hand & $14.1 \pm 4.1$ \\
Tip grip non-dominant hand & $13.8 \pm 4.0$ \\
\hline
\end{tabular}

Figures reported as mean \pm standard deviations. JTT $=$ Jebsen Taylor Test recorded in seconds, grip strength recorded as pounds per centimetre of pressure. 


\section{A. Dominant Hemisphere}

Total JTT

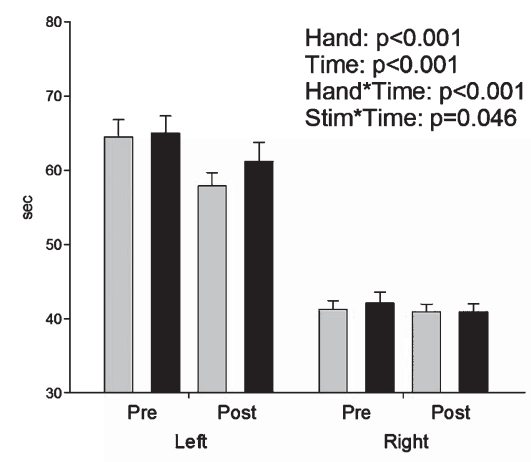

B. Non-Dominant Hemisphere

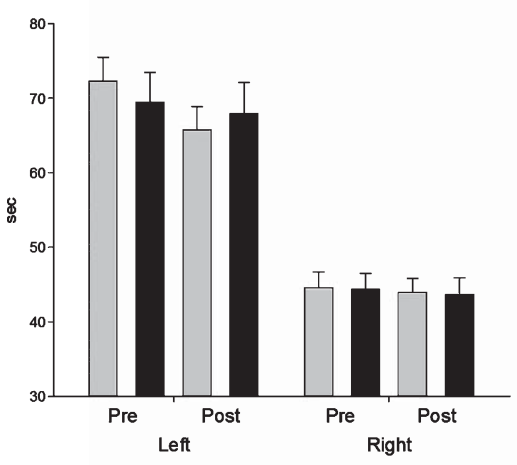

Fine Motor JTT
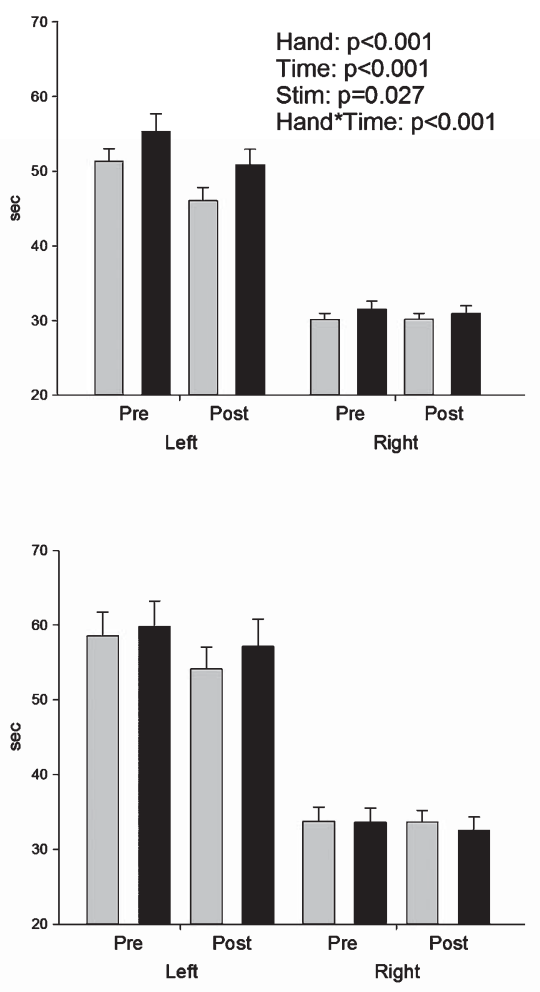

ACTIVE
Gross Motor JTT
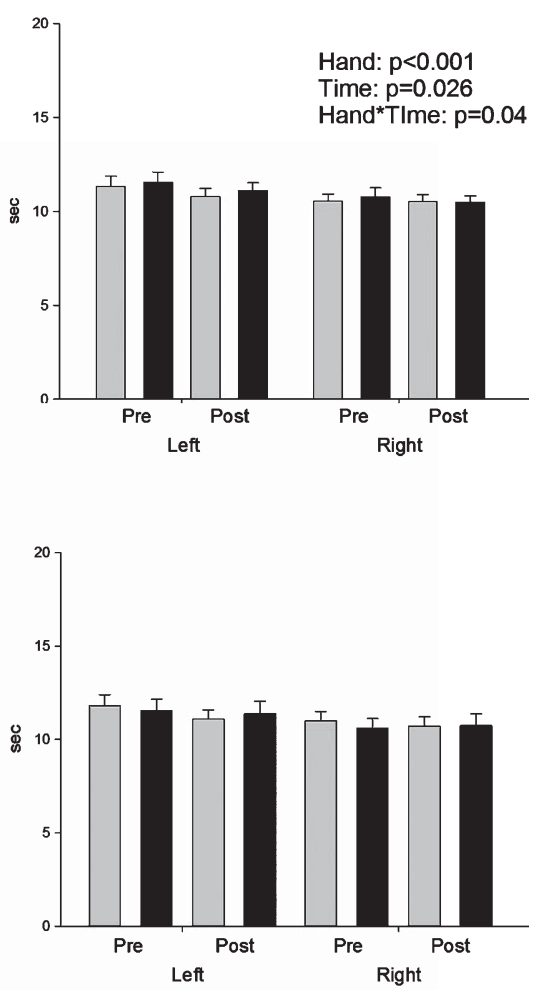

Fig. 1. Effects of (A) Dominant and (B) non dominant hemisphere stimulation on functional performance. Time (seconds) to complete total 7 items of Jebsen Taylor Hand Junction test (total JTT), fine motor items of JTT and gross motor items of JTT, pre and post stimulation.

advantage was evident regardless of whether stimulation was over the left or the right hemisphere. This is supported by the absence of any significant Hemisphere main effect or interaction. Age significantly affected total JTT score $(F(1,31)=6.3$, $p=0.017)$, but did not significantly mediate the size of the Stimulation $\times$ Time effect.

Fine motor JTT: Fig. 1 (centre) shows that fine motor JTT scores produced results compatible with those of the total JTT score. As above, responding was faster for right than left hand responses $(F(1,32)=407, p<0.001)$ and post-intervention compared to pre-intervention $(F(1,32)=20.1, p<0.001)$. The improvement from pre- to post-intervention was again greater for left than for right hand responses $(F(1,32)=17.8, p<0.001)$. There was a significant main effect of Stimulation
$(F(1,32)=5.34, p=0.027)$ and an interaction between Stimulation and Hand $(F(1,32)=6.47, p=0.016)$. Although there was no Stimulation $\times$ Time interaction, the data in Fig. 1 (centre) suggest that, like total JTT, stimulation improved performance for the left hand. Again, there was noeffect of Hemisphere of stimulation.

Gross motor JTT: As shown in Fig. 1 (right), gross JTT was faster for right than left hand $(F(1,32)=20.8, p<0.001)$, post-intervention compared to pre-intervention $(\mathrm{F}(1,32)=5.5, p=0.026)$, and this practice effect was greater for left than right hand responses $(\mathrm{F}(1,32)=4.6, p=0.04)$. However, there was no effect of Stimulation or Hemisphere.

Grip measures: Grip measure scores are shown in Fig. 2. There was no stimulation $\times$ time interaction on either measure. 


\section{A. Dominant Hemisphere}
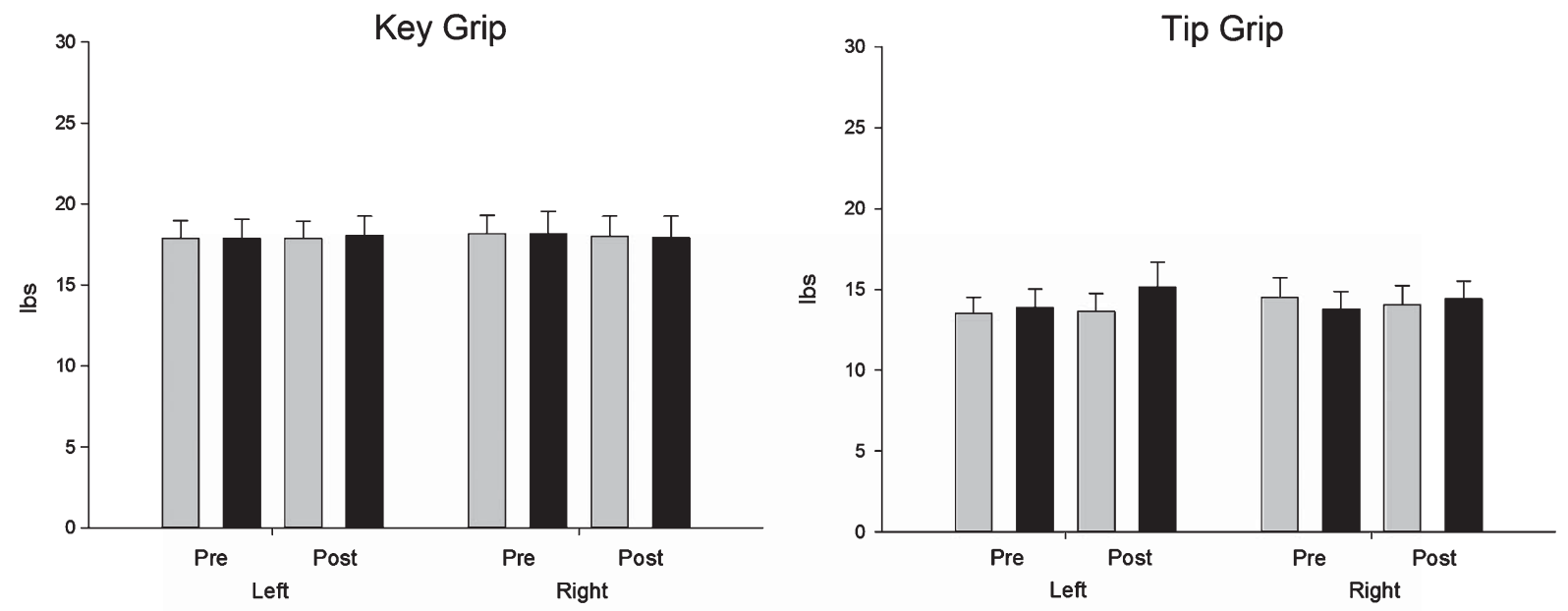

\section{B. Non-Dominant Hemisphere}
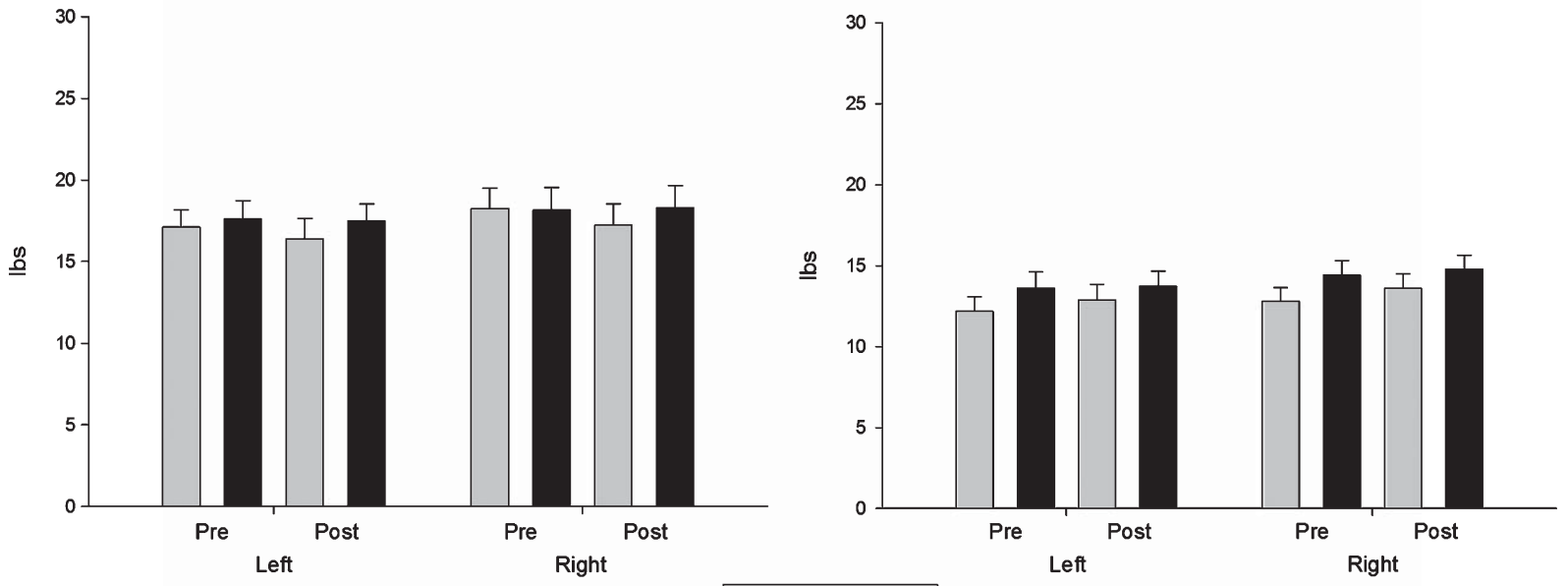

\section{ACTIVE}

Fig. 2. Effects of (A) dominant and (B) non dominant hemisphere stimulation on grip strength. Force (pounds per centimetre of pressure, lbs) exerted using key grip and tip pinch grip, pre and post stimulation.

\subsection{Go/Nogo task behavioural results}

Both dominant and non-dominant hemisphere stimulation groups responded faster to directional compared to non-directional cues $(F(1,32)=153.7$, $p<0.001)$ consistent with use of cues to prepare a motor response. As evident in Fig. 3, anodal tDCS stimulation did not reduce reaction time $(p>0.2)$. In fact, for the dominant hemisphere stimulation group, stimulation appears to have increased reaction time, especially for directional cues. This is shown in the significant interaction between stimulation, cue and hemisphere group $(F(1,32)=6.99, p=0.013)$.

\subsection{Electrophysiological results}

The electrophysiological data of two participants were removed from the analysis: one because of a 


\section{Dominant}

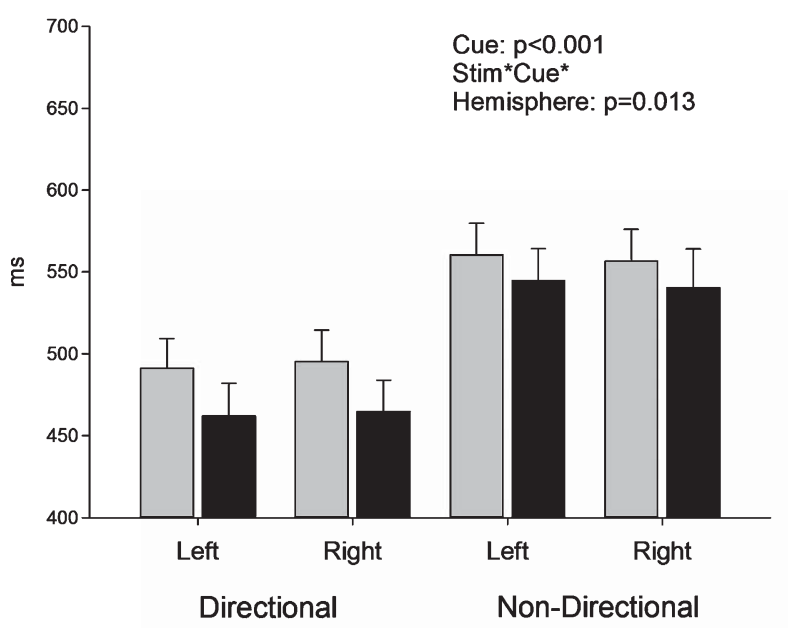

Non-Dominant

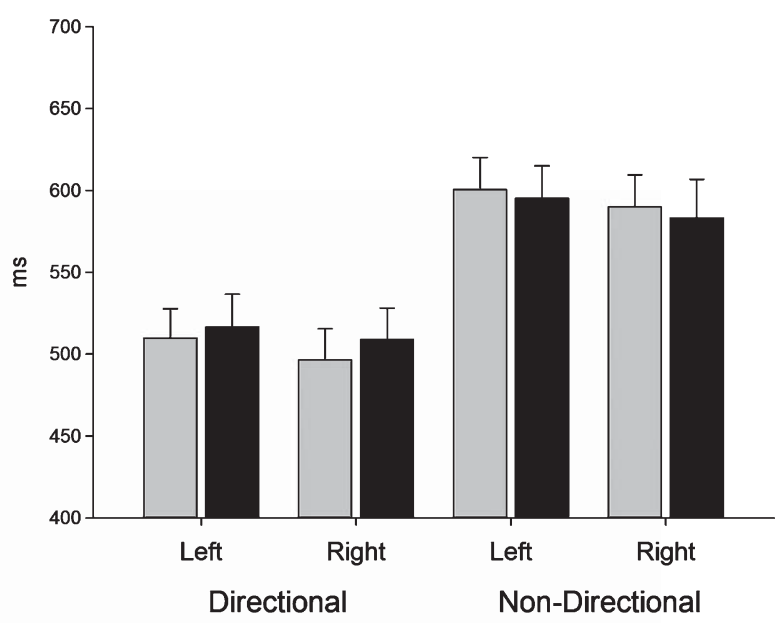

ACTIVE

SHAM

Fig. 3. Reaction time (sham - active stimulation). Difference in reaction times between sham and active conditions (time in milliseconds) in response to directional and non directional cues.

high level of artefact and the other because of a technical problem resulting in loss of data. Therefore, ERP analyses were completed on the remaining 32 participants. CNV amplitude was larger for directional than non-directional cues $(F(1,30)=8.96, p=0.005)$, indicating successful preparation in anticipation of target onset. Consistent with no behavioural effect of anodal tDCS on reaction time, anodal tDCS did not affect CNV amplitude $(F<1)$.

\subsection{Participant tolerance}

Participants reported mild and temporary sensory effects which were equivalent for the sham and stimulation sessions. There were no adverse reactions and no drop outs from the study.

\section{Discussion}

\subsection{Major findings}

The principal finding of this study was that a single session of anodal tDCS over the motor cortex of healthy aged subjects resulted in improved functional performance of fine motor tasks of the non-dominant hand irrespective of whether it was the dominant or non-dominant cortex which was stimulated. As anticipated, the dominant hand responded faster in all tasks however its performance did not improve with anodal tDCS. Electrophysiologically participants elicited larger CNV amplitudes for directional compared to non-directional cues however there was no beneficial effect of anodal tDCS on reaction times or response preparation on the go/no go task.

We anticipated improved performance of the contralateral hand with anodal stimulation. This was not observed with the dominant hand/cortex. This asymmetry in response to cortical stimulation has previously been observed in young subjects (Boggio, Castro et al., 2006, Vines, Nair et al., 2006, Williams, Pascual-Leone et al., 2010) and may reflect asymmetrical use of the hemispheres whereby the reduced dexterity and use of the non-dominant hand leads to relatively decreased cortical excitability of the non-dominant motor cortex (De Gennaro, Cristiani et al., 2004). The lack of effects in the dominant hand may represent a ceiling effect given that the dominant hemisphere is already optimally activated therefore increasing the excitability of this region with tDCS would infer no additional benefit on function (Zimerman and Hummel, 2010). This is supported by the findings of Furuya et al. (2014) who 
found that tDCS improved skilled finger movements in novice subjects but not in trained pianists indicating that functional changes in the motor cortex are dependent on the level of the expertise required for the task. Similarly it may reflect a ceiling effect of the assessment task itself which was relatively simple. In contrast there was statistically significant improvement in nondominant hand function. TMS studies have shown that the non-dominant cortex has a higher motor threshold suggesting tDCS may represent an effective way to lower the threshold, increase excitability and therefore hand performance (De Gennaro, Cristiani et al., 2004).

Our findings are in conflict with previous work in older adults which reported improved performance of the dominant hand with dominant hemisphere stimulation. This may be due to our sample being on average 9 years younger and potentially less impaired thus having less scope for measureable improvement than the participants of the Hummel et al., 2010 study; or due to the more complex nature of the task used by Zimerman et al. (2013) where a finger tapping sequence was used. Our study supports the notion that there is a degree of task specificity in the effects of tDCS (Hummel, Heise et al., 2010) such that the benefits were more pronounced on the fine motor tasks of the JTT and not the gross motor tasks, and there was no measurable change in the measures of grip strength.

Task specificity of the effects of tDCS may in part explain the disparity between the functional task results and performance on the go/nogo task. While on the functional tasks, stimulation produced some improvement in non-dominant hand performance, on the cued go/nogo task, there was no evidence of a positive effect of stimulation. In fact, dominant hemisphere stimulation resulted in slower reaction time compared to sham. Although improved function is the ultimate goal of stimulation, functional performance is the cumulative effect of many processes and is only an indirect and non-specific measure of motor-related cortical excitability. In contrast, tasks such as the cued go/nogo task presented here can be used to dissect motor performance into its underlying processes, and examine the level at which stimulation affects motor output. Here we report two levels: the final outcome (RT) and the earliest evidence of motor preparation $(\mathrm{CNV})$. The $\mathrm{CNV}$ indicates the level of readiness to respond to a validly predicted target and has been linked to level of excitation in the supplementary motor area and primary motor cortex (Luck, 2005). On analysis of final outcome (RT) and motor preparation (CNV), the current findings indicate that, despite some evidence of non-specific enhancement of non-dominant hand response speed with both dominant and non-dominant cortex stimulation, neither stimulation condition improved motor preparation or response speed. This may also be due to the timing of the stimulation in relation to the timing of EEG recordings which commenced approximately 40 minutes after the stimulation due to the time required for the functional assessments and EEG set up. Therefore any excitability effects may have returned to baseline in this time, or the functional assessments may have negated the effects of the stimulation. Thirugnanasambandam et al. (2011) demonstrated that the effects of anodal tDCS were reduced when stimulation was followed by an isometric muscle contraction which was sustained for two minutes. Our assessment of grip strength may have produced the same negating effect however as the EEG task required different neuronal circuits to the grip task, and the effects of tDCS are thought to be network specific, (Abraham, Mason-Parker et al., 2001) this can only be speculated. Similarly, there is debate in the literature whether tDCS and task performance should occur sequentially or concurrently. Some authors report that behavioural facilitation only occurred when tDCS was applied during the task execution (Guleyupoglu, Schestatsky et al., 2013, Stagg \& Jayaram, 2011) yet others state that tDCS must be applied prior to the task (Fertonani, Rosini et al., 2010, Vallar \& Bolignini, 2011). The effect of timing on the application of tDCS and the measurement of the response clearly needs further examination.

Improvement in non-dominant hand performance with anodal tDCS of the dominant hemisphere was not anticipated. Due to transcallosal inhibition, it would be reasonable to expect that anodal stimulation may lead to decreased excitability of the contralateral cortex and result in a detrimental effect on performance of the ipsilateral hand. The fact that the reverse occurred with respect to dominant cortex stimulation suggests that the ipsilateral motor cortex may, in certain instances, be relevant for motor performance in the non-dominant hand. This may especially be the case in older adults, as functional neuroimaging studies have demonstrated that the ageing brain shows more diffuse activation with less lateralisation during unilateral functional movement than in the young brain (Cabeza, McIntosh et al., 1997). Hence it is possible that participants recruited additional networks from the dominant 
hemisphere to compensate for age-related functional impairment and that tDCS has the capacity to augment this in older adults.

\subsection{Limitations}

We aimed to evaluate the effects of anodal stimulation of the primary motor cortex. However motor skill acquisition is a complex process involving multiple brain areas including prefrontal structures. The anodal montage used, whereby both electrodes are placed on the scalp, may have produced unwanted effects under the reference electrode. That is, anodal tDCS of the motor cortex occurs concurrently with cathodal stimulation of the frontopolar cortex potentially causing widespread excitability changes (Lang, Siebner et al., 2005). Furthermore, we used relatively large electrodes $\left(35 \mathrm{~cm}^{2}\right)$ which cover not only the primary motor cortex but also the adjacent cortices reducing the focality of the stimulation. In particularly, stimulation of the premotor cortex cannot be excluded however to date the effects of stimulation in this region are few and inconsistent (Pavlova, Kuo et al., 2014). Although this is the most commonly adopted electrode montage, future studies using an extracephalic reference or smaller anode electrode may overcome this concern.

\subsection{Clinical implications}

Previous studies have neglected to measure the bilateral upper limb effects of tDCS and therefore overlooked the potential importance of the ipsilateral descending pathways for movement performance. Current stroke research studies apply cathodal stimulation (not anodal) to the intact hemisphere to decrease excitability of this region in order to decrease transcallosal inhibition to the lesioned hemisphere. If we infer from our results that differences in the performance of the dominant and non-dominant hand reflect to some degree the differences between the paretic and non-paretic hands of stroke patients, our results would advocate the use of anodal stimulation to the intact hemisphere. This would seem particularly pertinent in the case of severe cortical stroke whereby the ipsilesional tracts may be the only intact descending pathway from the cortex. A neurophysiological model of ipsilateral limb control in stroke has recently been proposed (Bradnam, Stinear et al., 2013) and warrants further investigation.

\subsection{Conclusion}

A large body of tDCS research has focused on healthy young adults. This is a fundamental limitation as the main recipients of tDCS in the clinical setting are likely to be much older. There are considerable discrepancies regarding the effects of anodal tDCS on motor performance. This may be due to the nature of the task, the outcome measured, or multiple physical and anatomical differences between subjects. This study is unique in the breadth of examination to include both hemispheres and both upper limbs and demonstrated that the two hemispheres respond differently to anodal stimulation. This has established the foundations for subsequent comparisons between healthy aged subjects and patients with prevalent disease conditions such as stroke.

\section{Acknowledgments}

We extend our gratitude to our participants and the Hunter Medical Research Institute Volunteer Register for their assistance with advertising and recruitment.

No funding was sought for this project.

\section{Disclosure statement}

The authors have no conflicts of interest to disclose. The study was approved by the ethics committee of Hunter New England Health and ratified by the University of Newcastle (Ref: 10/11/17/4.02). All participants gave their written informed consent. The study was conducted according to the declaration of Helsinki Principles.

\section{References}

Abraham, W.C., Mason-Parker, S.E., Bear, M.F., Webb, S., \& Tate, W.P. (2001). Heterosynaptic metaplasticity in the hippocampus in vivo: A BCM-like modifiable threshold for LTP. Proc Natl Acad Sci USA, 98(19), 10924-10929.

Antal, A., Kincses, Z.T., Nitsche, M.A., Bartfai, O., \& Paulus, W. (2004). Excitability changes induced in the human primary visual cortex by transcranial direct current stimulation: Direct electrophyiological evidence. Invest Ophthalmol Vis Sci, 45, 702-707.

Antal, A., Terney, D., Poreisz, C., \& Paulus, W. (2007). Towards unravelling task-related modulations of neuroplastic changes induced in the human motor cortex. Eur J Neurosci, 26(9), 2687-2691. 
Bennett, K.M.B., \& Castiello, U. (1995). Reorganisation of prehension components following perturbation of object size. Psychol Aging, 10(2), 204-214.

Boggio, P.S., Castro, L.O., Savagim, E.A., Braite, R., Cruz, V.C., Rocha, R.R., Rigonatti, S.P., Silva, M.T., \& Fregni, F. (2006). Enhancement of non-dominant hand motor function by anodal transcranial direct current stimulation. Neurosci Lett, 404, 232-236.

Bradnam, L.V., Stinear, C.M., \& Byblow, W.D. (2013). Ipsilateral motor pathways after stroke: Implications for non-invasive brain stimulation. Front Hum Neurosci, 7, 184.

Burke, S.N., \& Barnes, C.A. (2006). Neural plasticity in the ageing brain. Nat Rev Neurosci, 7, 30-40.

Cabeza, R., McIntosh, A.R., Tulving, E., Nyberg, L., \& Grady, C.L. (1997). Age-related differences in effective neural connectivity during encoding and recall. Neuroreport, 8, 3479-3483.

Cogiamanian, F., Marceglia, S., Ardolino, G., Barbieri, S., \& Priori, A. (2007). Improved isometric force endurance after transcranial direct current stimulation over the human motor cortical areas. Eur J Neurosci, 26, 242-249.

De Gennaro, L., Cristiani, R., Bertini, M., Curcio, G., Ferrara M., Fratello, F., Romei, V., \& Rossini, P.M. (2004). Handedness is mainly associated with an asymmetry of corticospinal excitability and not of transcallosal inhibition. Clin Neurophysiol, 115(6), 1305-1312.

Dragovic, M. (2004). Towards an improved measure of the Edinburgh Handedness Inventory: A one-factor congeneric measurement model using confirmatory factor analysis. Laterality, 9(4), 411-419.

Fertonani, A., Rosini, S., Cotelli, M., Rossini, P.M., \& Miniussi, C. (2010). Naming facilitation induced by transcranial direct current stimulation. Behav Brain Res, 208(2), 311-318.

Floel, A., \& Cohen, L. (2010). Recovery of function in humans: Cortical stimulation and pharmacological treatments after stroke. Neurobiol Dis, 37(2), 243-251.

Furuya, S., Klaus, M., Nitsche, M.A., Paulus, W., \& Altenmuller, E. (2014). Ceiling effects prevent further improvements of transcranial stimulation in skilled musicians. J Neurosc, 34(41), 13834-13839.

Gandiga, P.C., Hummel, F., \& Cohen, L. (2006). Transcranial DC stimulation (tDCS): A tool for double-blind sham controlled clinical studies in brain stimulation. Clin Neurophysiol, 117, 845-850.

Guleyupoglu, B., Schestatsky, P., Edwards, D., Fregni, F., \& Bikson, M. (2013). Classification of methods in transcranial electrical stimulation (tES) and evolving strategy from historical approaches to contemporary innovations. J Neurosci Methods, 219(2), 297-311.

Herwig, U., Satrapi, P., \& Schonfeldt-Lecuona, C. (2003). EEG system for positioning of transcranial magnetic stimulation. Brain Topogr, 16, 95-99.

Hummel, F., Celnik, P., Giraux, P., Floel, A., Wu, W.H., Gerloff, C., \& Cohen, L.G. (2005). Effects of non-invasive cortical stimulation on skilled motor function in chronic stroke. Brain, 128, 490-499.
Hummel, F., Heise, K., Celnik, P., Floel, A., Gerloff, C., \& Cohen, L. (2010). Facilitating skilled right hand motor function in older subjects by anodal polarization over the left primary motor cortex. Neurobiol Aging, 31, 2160-2168.

Jebsen, R.H., Taylor, N., Trieschmann, R.B., Trotter, M.J., \& Howard, L.A. (1069). An objective and standardized test of hand function. Arch Phys Med Rehab, 50, 311-319.

Kang, E.K., \& and Paik, N.J. (2011). Effect of a tDCS electrode montage on implicit motor sequence learning in healthy subjects. Exp Transl Stroke Med, 3(4), 1-6.

Kincses, Z.T., Antal, A., Nitsche, M.A., Bartfai, O., \& Paulus, W. (2004). Facilitation of probabilistic classification learning by transcranial direct current stimulation of the prefrontal cortex in the human. Neuropsychologia, 42, 113-117.

Lang, N., Siebner, H.R., Ward, N.S., Lee, L., Nitsche, M.A., Paulus, W., Rothwell, J.C., Lemon, R.N., \& Frackowiak, R.S. (2005). How does transcranial DC stimulation of the primary motor cortex alter regional neuronal activity in the human brain? Eur J Neurosci, 22(2), 495-504.

Leuthold, H., Sommer, W., \& Ulrich, R. (2004). Preparing for action: Inferences from CNV and LRP. J Psycholphysiol, 18, 77-88.

Lu, T., Pan, Y., Kao, S., Li, C., Kohane, I., Chan, J., \& Yankner, B.A. (2004). Gene regulation and DNA damage in the ageing human brain. Nature, 429, 883-891.

Luck, S.J. (2005). An Introduction to the Event-Related Technique. Cambridge: Massachusetts Institute of Technology.

Nasreddine, Z., Phillips, N.A., \& Bedirian, V. (2005). The Montreal Cognitive Assessment, MoCA: A brief screening tool for mild cognitive impairment. J Am Geriatr Soc, 53, 695-699.

Nitsche, M.A., Cohen, L., Wassermann, E.M., Priori, A., Lang, N., Antal, A., Paulus, W., Hummel, F., Boggio, P.S., Fregni, F., \& Pascual-Leone, A. (2008). Transcranial direct current stimulation: State of the art 2008. Brain Stimul, 1, 206-223.

Nitsche, M.A., Schauenburg, A., Lang, N., Liebetanz, D., Exner, C., \& Paulus, W. (2003). Facilitation of implicit motor learning by weak transcranial direct current stimulation of the primary motor cortex in the human brain. J Cognitive Neurosci, 15, 619-626.

Oliviero, A., Profice, P., Tonali, P.A., Pilato, F., Saturno, E., Dileone, M., Ranieri, F., \& Di Lazarro, V. (2006). Effects of ageing on motor cortex excitability. Neurosci Res, 55, 74-77.

Ozcan, A., Tulum, Z., Pinar, L., \& Baskurt, F. (2004). Comparison of pressure pain threshold, grip strength, dexterity and touch pressure of dominant and non dominant hands within and between right and left handed subjects. J Korean Med Sci, 19, 874-878.

Pavlova, E., Kuo, M., Nitsche, M., \& Borg, J. (2014). Transcranial direct current stimulation of the premotor cortex: Effects on hand dexterity. Brain Res, 1576, 52-62.

Quartarone, A., Morgante, F., Bagnato, S., Rizzo, V., Sant'Angelo, A., Aiello, E., Reggio, E., Battaglia, F., Messina, C., \& Girlanda, P. (2004). Long lasting effects of transcranial direct current stimulation on motor imagery. Neuroreport, 15(8), 1287-1291. 
Reis, J., Schambra, H.M., Cohen, L., Buch, E.R., Fritsch, B., Zarahn, E., Celnik, P.A., \& Krakauer, J.W. (2009). Noninvasive cortical stimulation enhances motor skill acquisition over multiple days through an effect on consolidation. Proc Natl Acad Sci USA, 106(5), 1590-1595.

Spreng, R.N., Wojtowicz, M., \& Grady, C.L. (2010). Reliable differences in brain activity between young adults: A quantitative meta-analysis across multiple cognitive domains. Neurosci Biobehav R, 34(8), 1178-1194.

Stagg, C.J., Jayaramm, G., Pastor, D., Kincses, Z.T., Matthews, P.M., \& Johansen-Berg, H. (2011). Polarity and timing-dependent effects of transcranial direct current stimulation in explicit motor learning. Neuropsychologia, 49, 800-804.

Tecchio, F., Zappasodi, F., Assenza, G., Tombini, M., Vollaro, S., Barbati, G., \& and Rossini, P.M. (2010). Anodal transcranial direct current stimulation enhances procedural consolidation. $J$ Neurophysiol, 104, 1134-1140.

Thirugnanasambandam, N., Sparing, R., Dafotakis, M., Meister, I.G., Paulus, W., Nitsche, M.A., \& Fink, G.R. (2011). Isometric contraction interferes with transcranial direct current stimulation (tDCS) induced plasticity: Evidence of state-dependent neuromodulation in human motor cortex. Restor Neurol Neurosci, 29(5), 311-320.

Vallar, G., \& Bolognini, N. (2010). Behavioural facilitation following brain stimulation: Implications for neurorehabilitation. Neuropsychol Rehabil, 21(5), 618-649.
Vines, B.W., Cerruti, C., \& Schlaug, G. (2008). Dual-hemisphere tDCS facilitates greater improvements for healthy subjects' non-dominant hand compared to uni-hemisphere stimulation. BMC Neurosci, 9, 103.

Vines, B.W., Nair, D.G., \& Shlaug, G. (2006). Contralateral and ipsilateral motor effects after transcranial direct current stimulation. Neuroreport, 17(6), 671-674.

Williams, J.A., Pascual-Leone, A., \& Fregni, F. (2010). Interhemispheric modulation induced by cortical stimulation and motor training. Phys Ther, 90(3), 398-410.

Zaehle, T., Sandmann, P., Thorne, J., Jancke, L., \& Herrmann, C. (2011). Transcranial direct current stimulation of the prefrontal cortex modulates working memory performance: Combined behavioural and electrophysiological evidence. BMC Neurosci, 12, 1-11.

Zimerman, M., \& Hummel, F. (2010). Non-invasive brain stimulation: Enhancing motor and cognitive functions in healthy old subjects. Front Aging NeuroscI, 2, 149.

Zimerman, M., Nitsch, M., Giraux, P., Gerloff, C., Cohen, L.G., \& Hummel, F.C. (2013). Neuroenhancement of the ageing brain: Restoring skill acquisition in old subjects. Ann Neurol, 73, 10-15. 